\title{
Integração de uma Metodologia de Ensino Presencial de Programação com um Sistema Tutor Inteligente
}

\author{
Integration of a Methodology for Teaching Programming with an Intelligent Tutoring \\ System.
}

Sahudy Montenegro González

Universidade Federal de São Carlos (UFSCar),

Campus Sorocaba, SP - Brasil

sahudy@ufscar.br

\author{
Annabell del Real Tamariz \\ Universidade Estadual do Norte Fluminense (UENF) \\ Campos dos Goytacazes, RJ - Brasil \\ annabell@uenf.br
}

\begin{abstract}
Resumo O presente trabalho propõe a aplicação de uma metodologia de ensino de programação, integrada à utilização de recursos tecnológicos como instrumento de apoio ao ensino presencial. Assim, foi desenvolvido um ambiente virtual, denominado O HALYEN, como um Sistema Tutor Inteligente, baseado em agentes inteligentes. O propósito do ambiente é atuar como uma ferramenta tecnológica de auxílio ao ensino presencial, através da escolha dinâmica da estratégia pedagógica, segundo o perfil, estado emocional e outras características de cada aluno. Este artigo apresenta a metodologia utilizada em sala de aula para o ensino de programação e foca na descrição do ambiente virtual. Como parte do ambiente inteligente foram definidas quatro emoções. Essas emoções são inferidas a partir da definição de eventos, regras e um perfil inicial do aluno calculado com base na aplicação do questionário MSLQ. Os resultados preliminares da proposta se mostram bastante animadores e vêm da execução de uma primeira oficina com alunos do curso de Computação para coleta de dados para o treinamento da inferência das emoções e a avaliação das estratégias pedagógicas propostas.
\end{abstract}

Palavras-Chave: Ensino de programação, sistema tutor inteligente, agentes inteligentes, jogos de lógica, inferência de emoções, MSLQ, modelo do aluno, estratégia pedagógica.

\begin{abstract}
This work proposes to apply a methodology for teaching programming integrating technology resources to support classroom teaching. Thus, we developed a virtual environment, called $O$ HALYEN as an Intelligent Tutoring System based on intelligent agents. The purpose of the environment is to act as a technological tool to aid classroom learning through dynamic selection of the teaching strategy, according to the profile, emotional state and other characteristics of each student. This article presents the methodology used in the classroom for teaching programming and focuses on the system specifics. As part of the intelligent system four emotions were defined. These emotions are inferred from the definition of events, rules and a MSLQ-based initial profile of the student. The preliminary results show the proposal is very promising since a first workshop was run to collect training data for emotion inference and evaluation of the proposed pedagogical strategies.
\end{abstract}

Keywords: Programming learning, intelligent tutoring system, intelligent agents, logic games, emotion inference, MSLQ, student model, pedagogical strategy. 


\section{Introdução}

O ensino de programação está regularmente presente nas grades curriculares dos cursos de graduação relacionados a Engenharias e Computação. Segundo uma pesquisa desenvolvida pelos autores de [1] e constatada nos trabalhos [2,3], as disciplinas relacionadas ao ensino de programação são as que apresentam maior índice de reprovação e o seu ensino é considerado um grande desafio. Muitas pesquisas, dentre elas $[4,5,6]$, propõem a utilização de ferramentas para auxílio ao ensino de programação, motivados por essas circunstâncias.

Para corroborar essas pesquisas, as autoras realizaram um levantamento enquanto ministravam disciplinas relacionadas ao ensino de programação. As disciplinas foram lecionadas no primeiro período dos cursos de Engenharia de Produção e Ciência da Computação. Dentre os 175 alunos observados, durante quatro semestres, cerca de $54 \%$ foram reprovados, seja por desistência ou por rendimento abaixo da nota mínima de aprovação [7]. Este índice de reprovações é considerado alto e constitui o principal motivo para o desenvolvimento deste trabalho.

Devido à situação apresentada, decidiu-se aplicar a metodologia de ensino de programação descrita em [8]. Como mostra o trabalho descrito em [7], após um ano de trabalho em sala de aula, aplicando somente esta metodologia, puderam-se constatar bons resultados na sua aplicação para alunos que, apesar de não estudar computação (ou cursos afins), precisam aprender programação como parte de integralização de seus créditos.

Com o propósito de continuar aprimorando esta abordagem, levantaram-se as questões: A inclusão de recursos tecnológicos no ambiente de aprendizagem é efetiva para auxiliar o trabalho em sala de aula? A inferência das emoções dos alunos utilizando recursos tecnológicos no ambiente de aprendizagem pode influenciar positivamente no aprendizado dos alunos?

Como apoio teórico à primeira questão, [9] analisa a relação entre sujeito e objeto e como através desse processo de interação, as reações do objeto às ações do sujeito permitem construir modelos mentais que ganham importância e se convertem em uma parte fundamental do processo de aprendizagem. Em [10], os autores apresentam a utilização de um agente animado, como recurso tecnológico interessante e motivador, para o aprendizado no ensino fundamental.

A respeito da segunda questão, alguns estudiosos já afirmaram que o estado emocional de um aluno tem influência na sua capacidade de aprendizagem. Nesse sentido, as condições afetivas positivas favorecem o processo de aprendizagem [11]. Reforçando essa ideia, a pesquisa descrita em [12] envolveu um grupo de alunos em níveis diferentes de ensino e revelou que "as ações mediadoras dos professores criam situações que produzem diversos sentimentos e emoções que influenciam o processo ensino-aprendizagem".

O presente trabalho propõe a aplicação da metodologia de ensino de programação descrita em [8], integrada à utilização de recursos tecnológicos como instrumento de apoio ao ensino presencial. Assim, foi desenvolvido um sistema virtual, denominado O HALYEN, como um Sistema Tutor Inteligente (STI). O propósito do ambiente é atuar como uma ferramenta de auxílio ao ensino presencial, através da escolha dinâmica da estratégia pedagógica, segundo a avaliação inferida do perfil e emoções de cada aluno. O foco tanto da metodologia quanto do ambiente é a aprendizagem baseada em jogos computacionais.

A Seção 2 deste artigo descreve os trabalhos relacionados ao ensino de programação com apoio de tecnologias computacionais. Na Seção 3, foca-se na arquitetura do ambiente O HALYEN e as fases propostas. A Seção 4 descreve o modelo afetivo e a determinação do perfil do aluno dentro do ambiente. A Seção 5 explica a aplicação da metodologia para o ensino de programação e a utilização do ambiente como instrumento de apoio à mesma. $\mathrm{Na}$ Seção 6 definem-se os eventos e as regras de inferência das emoções. Na Seção 7, apresentam-se os resultados da integração da metodologia com o ambiente computacional. A Seção 8 expõe as considerações finais do trabalho.

\section{Contextualização e Trabalhos Rela- cionados}

No caso específico do estudo de computação, o grande desafio consiste em desenvolver habilidades relacionadas ao raciocínio lógico-matemático. Desenvolver tal capacidade nos alunos é uma árdua tarefa, que vem causando desânimo por parte dos alunos quando enfrentam o estudo desta disciplina, favorecendo a ocorrência de reprovações e desistências. Neste contexto, o desafio do professor é procurar desenvolver em sala de aula uma maneira de criar habilidades de raciocínio lógicomatemático dos alunos.

Adicionalmente, a inferência das emoções do aluno durante o desenvolvimento de uma atividade é um assunto difícil de ser tratado computacionalmente, pois as emoções e desejos de cada aluno devem ser modelados para saber como o aluno está se sentindo com respeito à matéria cursada. Por exemplo, se um aluno está jogando como parte de uma atividade e fica frustrado por não conseguir ganhar o jogo, ele provavelmente irá desistir desse jogo, e mais importante, de tentar entender como ganhar. O sistema precisa saber quando o aluno está frustrado para incentivá-lo a jogar novamente. 
Um ambiente de aprendizagem baseado em um STI deve ser flexível e permitir a interação com o aluno [13, 14]. Na interação, o ambiente poderá ser capaz de "modelar" o aluno e, assim, aplicar diferentes estratégias de ensino. Na literatura recente existem várias propostas que desenvolvem STIs. Os trabalhos em [15], [16] e [17] propõem o uso de tutores inteligentes para o ensino de álgebra, controladores lógicos programáveis e para a construção de modelos matemáticos de sistemas dinâmi$\cos$, respectivamente. Especificamente para o ensino de programação, [18] integra o modelo do aluno proposto em um ambiente educacional Web para o ensino de programação em C. O modelo do aluno é atualizado de acordo com uma técnica de lógica fuzzy, que descreve o nível de conhecimento do aluno para cada conceito do domínio. Em [19], é descrito DEPTHS (Design Patterns Teaching Help System), um STI para o ensino de padrões de projeto. A adaptação do material escolhido para o ensino baseia-se no desempenho do aluno e em comportamentos coletados durante as sessões de estudo. Em relação a abordagens que integram emoções e jogos computacionais, o estudo descrito em [20] trabalha com a hipótese de influenciar a cognição e a aprendizagem através de um conjunto de estados cognitivo-afetivos como são o tédio, a confusão, a alegria, a surpresa, a concentração e a frustração.

Várias propostas tiram proveito do uso de jogos, ferramentas colaborativas e/ou ambientes inteligentes para desenvolver estratégias de ensino/aprendizagem. Dentre eles, podem ser citados alguns trabalhos relacionados ao ensino de linguagens de programação como [9], [21], [22], [23], [24], [25] e [26].

A presente proposta integra o uso de ferramentas computacionais em um ambiente inteligente com a aplicação de uma metodologia de ensino na sala de aula. Os resultados obtidos e apresentados em [6] serviram de motivação para a utilização dessa última. Como a metodologia de ensino é baseada em jogos computacionais, o ambiente oferece esse suporte. Adicionalmente, O HALYEN provê suporte para determinar o perfil do aluno, inferir suas emoções e escolher a estratégia pedagógica mais adequada.

\section{O HALYEN}

O sistema O HALYEN foi desenvolvido para auxiliar à aplicação da metodologia descrita em [6]. O objetivo da metodologia é oferecer uma abordagem pedagógica para a iniciação ao estudo de algoritmos e programação. A mesma está dividida em três passos, que serão detalhados na Seção 5.

O sistema O HALYEN é um ambiente virtual e um STI, concebido para servir de suporte nas diferentes fases da metodologia e tentar capturar o estado emocional dos alunos. O ambiente possui duas fases de execução, explicadas na Seção 3.3. O sistema tem três tipos de usuários: administrador, professor e aluno. O administrador faz os cadastros de alunos, turmas, professores, disciplinas e cursos. O professor gerencia o conteúdo instrucional e é responsável pela configuração e acompanhamento do desempenho do aluno. Este efetua o preenchimento da base do domínio correspondente ao conteúdo da matéria que será passada ao aluno. Os alunos possuem acesso a materiais, à execução de exercícios e ao gerenciamento de dados pessoais.

A arquitetura conceitual do ambiente pode ser analisada por completo em [27, 28]. Conforme a arquitetura tradicional de um STI [14], a proposta contém os quatro componentes principais: o modelo de aluno, modelo de domínio, o modelo do tutor e a interface, explicados com maiores detalhes em [28, 29].

Em um ambiente Web, o conteúdo instrucional armazenado no modelo do domínio, pode ser formado por hipertextos, jogos e gráficos, dentre outros. Adicionalmente, são definidos atributos que possibilitam ao modelo do tutor tomar decisões referentes ao processo de acompanhamento do aluno. No modelo tutor, são definidas as estratégias pedagógicas ou ações que o sistema deve tomar considerando uma configuração específica do modelo do aluno e do domínio. As ações adotadas pelo tutor normalmente refletem o que é mais apropriado para o processo de aprendizagem, de acordo com a teoria pedagógica que sustenta as suas decisões.

\subsection{Visão geral}

Para facilitar o entendimento do funcionamento do ambiente, apresenta-se o passo a passo que o aluno segue ao entrar no mesmo. Destaca-se que o sistema tutor é uma ferramenta que auxilia no processo de aprendizagem presencial. Portanto, cada passo no ambiente tem uma contrapartida na sala de aula.

- Responde o questionário Motivated Strategies for Learning Questionnaire (MSLQ) (obrigatório na primeira vez ou sempre que ele requisitar).

- Resolução de cada jogo classificado como fácil.

- Exposição da resolução do problema e prosseguimento da metodologia na sala de aula.

- Resolução de cada jogo classificado como de nível médio.

- Exposição da resolução do problema e prosseguimento da metodologia na sala de aula.

- Resolução de cada jogo classificado como de nível difícil.

- Exposição da resolução do problema e seguimento da metodologia na sala de aula. 
- Utilização da interface de programação para especificação da solução dos problemas em forma de algoritmos (Fase 3 da metodologia)

Do ponto de vista de tutor inteligente, o sistema:

- Determina o perfil inicial do aluno, após a aplicação do MSLQ.

- Durante as atividades:

(i) infere o comportamento emocional (emoções do aluno), baseado no modelo de emoções (descrito na Seção 4) e nas ações do aluno;

(ii) determina as táticas pedagógicas aplicadas (utilizando o perfil e a emoção): auxílio cognitivo utilizando os assistentes (por exemplo, o assistente a dúvidas) e/ou ajuda emocional com mensagens de encorajamento.

\subsection{Agentes Inteligentes}

O sistema O HALYEN foi elaborado a partir de uma arquitetura de sistema baseada em agentes inteligentes, utilizando um sistema multiagente com agentes deliberativos. Optou-se por essa abordagem diante da necessidade de avaliar o aluno sob diversos aspectos. Além de medir o estado emocional do aluno ao interagir com o ambiente, considera-se importante avaliar melhor a motivação do aluno [27].

A arquitetura da Figura 1 apresenta os agentes inteligentes e descreve o fluxo de dados entre todos os elementos da arquitetura conceitual.
O agente aprendiz na Figura 1, é focado no modelo de emoções e no modelo cognitivo. Este agente interage com as bases desses modelos, de forma a obter/gravar informações referentes ao(s) aluno(s) e seus respectivos perfis e estados emocionais. O agente tutor interage com os agentes de domínio e aprendiz, sendo que, baseado nas informações recebidas, este avalia a melhor estratégia de ensino para aprimorar ou manter o estado emocional e nível de aprendizagem do aluno. O agente de domínio acessa a base de dados do modelo cognitivo, de forma a fornecer subsídios ao agente tutor, para que este faça a melhor escolha no que diz respeito a estratégia de ensino.

$\mathrm{O}$ agente assistente tem como objetivo integrar ao ambiente Assistentes Inteligentes de Ensino (ITAs - Intelligent Teaching Assistant systems) [30]. Ele auxilia os estudantes e assiste ao professor em suas tarefas. Assim, aumenta-se a interação do aluno com o ambiente. O mesmo, fica responsável por ativar os assistentes inteligentes de ensino. Inicialmente, foi disponibilizado o assistente de dúvidas aos alunos. O aluno pode pedir ajuda através da formulação de dúvidas na linguagem natural (português) e este assistente retorna a melhor resposta possível, auxiliando-se de uma base de respostas por matéria, preenchidas pelos professores especialistas [31]. A Figura 2 mostra a interface desse assistente. Este é ativado quando o aluno pede assistência ou o agente tutor achar necessário em algum ponto do processo de ensino/aprendizagem.

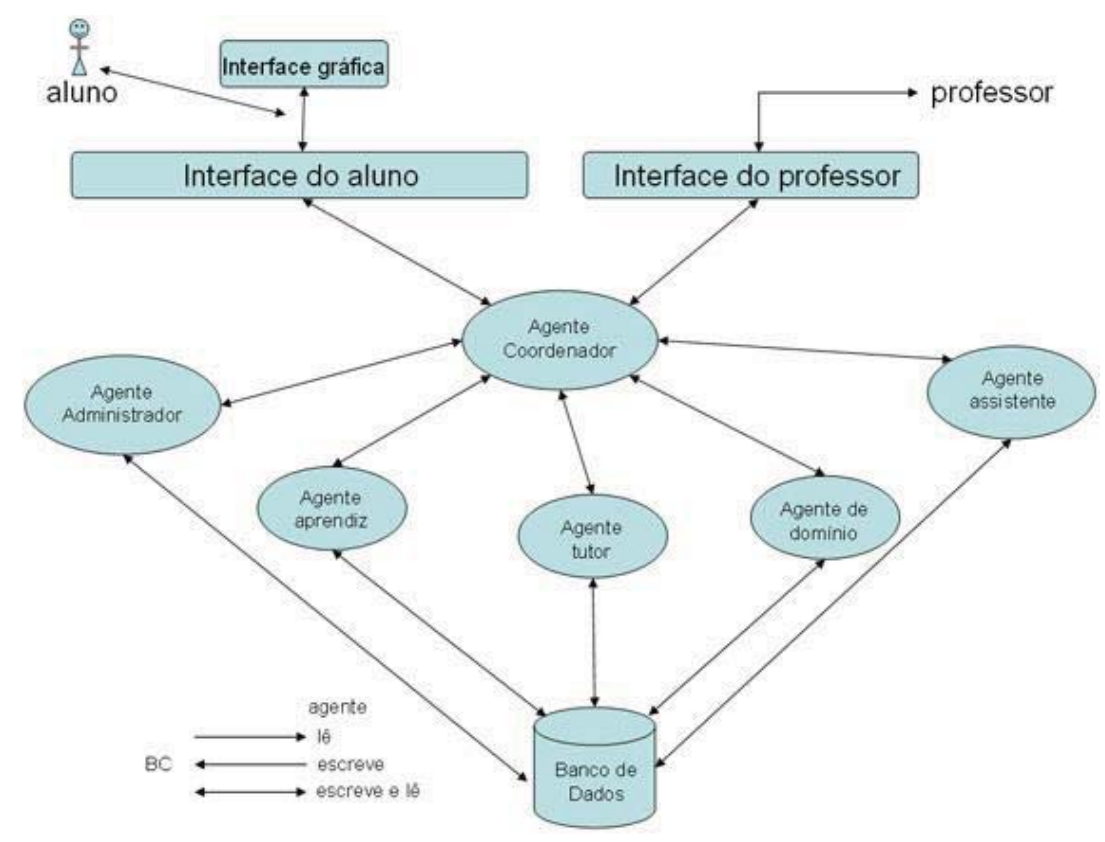

Figura 1: Arquitetura de O HALYEN baseada em agentes inteligentes 


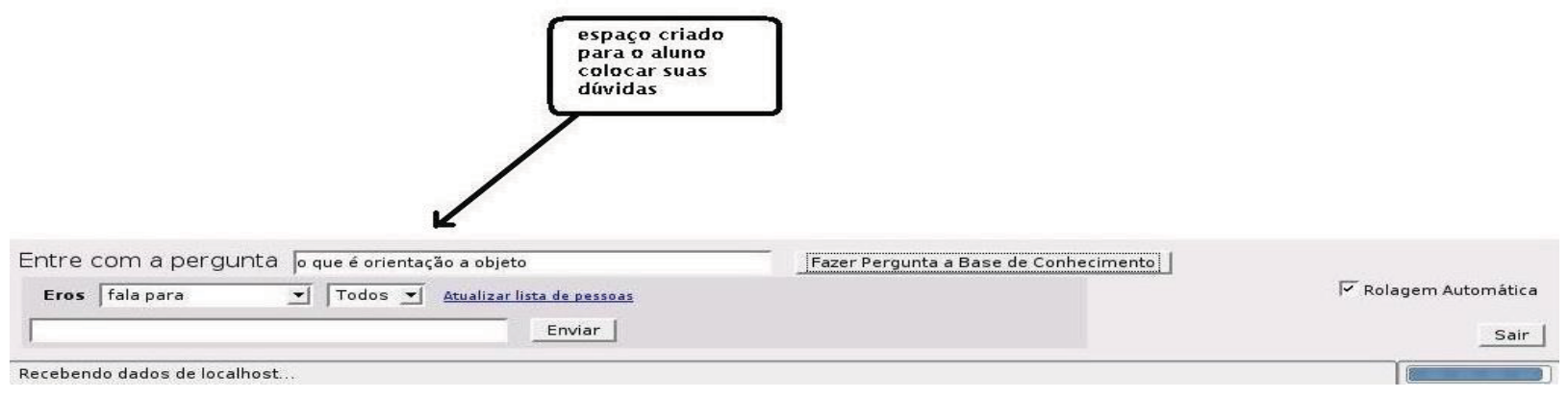

Figura 2: Interface do Assistente às dúvidas

A arquitetura acrescentou o agente coordenador, que realiza a coordenação das tarefas, recebe as requisições do ambiente e as encaminha para o agente capacitado para resolvê-las. A troca de informações entre os agentes permite que o aluno receba um conteúdo instrucional adaptado a sua necessidade por meio da interface do aluno. A interface do professor permite a interação entre o professor e as funcionalidades atribuídas ao mesmo.

\subsection{Fases do Ambiente}

O ambiente O HALYEN é definido para ser executado em duas fases: treinamento e aplicação.

O objetivo da fase de treinamento é "aprender". Esta primeira fase vai coletando dados sobre as diferentes interações dos alunos no ambiente. Esses dados permitem estabelecer a manifestação de uma determinada emoção e descobrir diferentes perfis de aluno. Espera-se que, estudando melhor o comportamento de grupos de alunos, possa-se escolher melhores estratégias de ensino para alunos com comportamento similar. Os dados são coletados em oficinas com alunos do primeiro período dos cursos de computação e afins. Os perfis e estratégias pedagógicas aprendidos com os dados coletados serão aplicados para acompanhar o aprendizado dos alunos quando da execução do ambiente na sua segunda fase.

Na primeira etapa, utilizam-se as bases de dados referentes ao aluno, pois é uma fase de coleção de dados de treinamento para posterior análise. O agente aprendiz armazena dados das interações dos alunos. Por exemplo, número de partidas executadas para resolver um jogo, número de movimentos em cada partida e a sequência de eventos executados em uma partida. Com esses dados, é possível caracterizar o aluno quanto ao desempenho nos exercícios e ao grau de conhecimento do tema. Outros atributos do aluno poderão complementar a análise de perfil, dentre eles, a avaliação inicial do perfil, que será apresentada na Seção 4; o grau de sociabilidade e o grau de empenho. Por exemplo, o grau de sociabilidade do aluno poderá ser capturado a partir da coleta de quais ferramentas ou assistentes do ambiente ele utiliza; e empenho pode ser determinado pela presença, isto é, o nú- mero de acessos, tempo de cada acesso e de execução de tarefas dentro do ambiente.

As características dos alunos podem definir perfis e padrões de emoções que permitem construir estratégias de ensino baseadas em teorias pedagógicas. Os dados coletados podem ser minerados utilizando técnicas descritivas, para reconhecer o tipo de perfil do estudante, mas não faz parte deste trabalho no momento.

Os padrões descobertos após a fase de treinamento, são utilizados para mediar o aprendizado de novos alunos no ambiente. Casos atípicos podem acontecer durante a fase de aplicação, que podem ser considerados para um novo perfil.

\section{Determinação do perfil do aluno}

Uma das características definidas para o ambiente $\mathrm{O}$ HALYEN é possibilitar a inferência das emoções do aluno baseando-se no comportamento observável, ou seja, através de interações do usuário com o ambiente [32]. O modelo OCC (Othony, Clore e Collins) [33] foi escolhido como alternativa adequada, e já vem sendo amplamente utilizado em aplicações [34,35,36,37], voltadas para o ensino à distância, ambientes de simulações de agentes com emoções, dentre outros.

O modelo OCC destaca que as emoções surgem quando eventos são avaliados de acordo com a desejabilidade em relação aos objetivos de uma pessoa. Para estabelecer os objetivos de um aprendiz, aplicou-se o questionário MSLQ [35,38]. O instrumento MSLQ foi desenvolvido por um grupo de pesquisadores em Psicologia e Educação da Universidade de Michigan. Um estudo recente [39] fez uma revisão de 363 estudos (levantados de artigos de periódicos e congressos, dissertações e outros relatórios) que aplicam o questionário MSLQ. No geral, os resultados mostram que as seções de motivação e estratégias de aprendizado podem ser aplicadas com resultados aceitáveis.

O ambiente considera duas categorias de estados de ânimo relacionados às emoções: estar animado e estar 
desanimado como proposto em [35]. Na primeira categoria, o aluno deve demonstrar um comportamento alegre. Motivação, interesse em enfrentar os desafios da aprendizagem e satisfação com os resultados atingidos são as características esperadas. Na segunda categoria, o aluno demonstra um comportamento triste. Desinteresse, desmotivação, insatisfação, frustração com os resultados alcançados e sentir-se penalizado são as características que podem ser deduzidas.

Essas quatro emoções destacadas (alegria, satisfação, tristeza e frustração) são consideradas perfeitamente compatíveis e possíveis de serem sentidas pelo aluno durante sua interação com o ambiente [40]. Na Seção 6, descrevem-se os eventos relacionados às emoções, definidos no escopo do ambiente.

\subsection{Perfil inicial}

No primeiro acesso do aluno ao ambiente, é apresentado o formulário que avalia sua motivação inicial. Na Tabela 1 apresentam-se as perguntas do questionário e sua relação com o perfil: orientado à aprendizagem $(O A)$ ou ao desempenho (OD). A motivação do aluno é analisada utilizando o questionário MSLQ, especificamente, as questões dedicadas às metas de orientação intrínseca e extrínseca, utilizadas também em [32,36,37]. Para determinar o perfil do aluno, cada pergunta no questionário tem uma pontuação definida, sendo que ao final, o aluno tem sua orientação definida a partir do somatório obtido no questionário [27].

\begin{tabular}{|l|l|}
\hline Pergunta & Orientação \\
\hline $\begin{array}{l}\text { Em uma classe como essa, eu prefiro um conteúdo que } \\
\text { realmente me desafia a aprender coisas novas. }\end{array}$ & Aprendizagem \\
\hline $\begin{array}{l}\text { Em uma classe como essa, eu prefiro um conteúdo que } \\
\text { aguça a minha curiosidade, mesmo que seja mais dificil } \\
\text { de aprender. }\end{array}$ & Aprendizagem \\
\hline $\begin{array}{l}\text { A coisa mais satisfatória para mim nesse curso é tentar } \\
\text { compreender o conteúdo tão completamente quanto } \\
\text { possivel. }\end{array}$ & Aprendizagem \\
\hline $\begin{array}{l}\text { Quando eu tiver oportunidade na classe, eu escolherei } \\
\text { uma tarefa do curso que eu possa aprender mesmo não } \\
\text { garantindo uma boa nota. }\end{array}$ & Aprendizagem \\
\hline $\begin{array}{l}\text { Tirar uma boa nota nessa classe é a coisa mais } \\
\text { satisfatória para mim nesse momento. }\end{array}$ & Desempenho \\
\hline $\begin{array}{l}\text { A coisa mais importante para mim nesse momento é } \\
\text { aumentar a minha média total de notas, sendo assim, } \\
\text { meu principal interessena classe é tirar uma boa nota. }\end{array}$ & Desempenho \\
\hline $\begin{array}{l}\text { Se eu puder, eu quero tirar melhores notas na classe do } \\
\text { que a maioria. }\end{array}$ & Desempenho \\
\hline $\begin{array}{l}\text { Eu quero sair-me bem na classe porque é importante } \\
\text { mostrar minha habilidade para minha familia, amigos; } \\
\text { patrão e outros. }\end{array}$ & Desempenho \\
\hline
\end{tabular}

Tabela 1: Questões do MSLQ relacionadas à orientação do aluno

As respostas ao questionário MSLQ são recolhidas para cada aluno e armazenadas no banco de dados.

\section{Aplicando a Metodologia}

$\mathrm{O}$ processo de interação entre o aluno e o ambiente permite a construção de modelos mentais que fazem parte do processo de aprendizagem [9]. Muitos dos esforços em melhorar a aprendizagem se apoiam na colaboração e troca entre os alunos. A metodologia aplicada neste artigo se insere no contexto de trabalho em pares. De acordo com Vygotsky (1991 apud [12]) é importante a interatividade entre o indivíduo e o meio social. Do ponto de vista pedagógico, isto justifica o trabalho colaborativo, possibilitando melhores resultados em comparação com o trabalho individual [22]. Trabalhar em pares (na primeira fase da metodologia), valendo-se de um instrumento mediador (o problema), implica no desenvolvimento da interação através da explicitação das estratégias, por parte do aluno, para a resolução das tarefas (a resolução do problema).

A metodologia foi aplicada nessa sequência:

1. Resolução de Problemas: (i) os alunos tentam achar a solução dos vários jogos de lógica; (ii) quando achada, para cada jogo, anotam os passos seguidos para chegar à solução; (iii) os alunos trocam cadernos em duplas com o objetivo de conferir e corrigir a solução dos colegas, visando aprimorar a solução por meio de uma escrita melhor formalizada.

2. Formalização: o professor continua com a resolução dos jogos, porém, a turma e o professor definem substantivos e verbos limitados para elaborar as soluções. Por exemplo, para o jogo da Torre de Hanói sugerem-se seis substantivos (pinos e peças) e os verbos: mover; voltar.

3. Construção de Algoritmos: (i) construção de algoritmos em português, destacando a entrada de dados, o processamento e a saída do algoritmo. Definem-se as instruções ou comandos por meio de palavras. (ii) Treinamento com exercícios de construção de algoritmos. (iii) Mapeamento dos comandos dos algoritmos para instruções na linguagem de programação. (iv) Exercícios no caderno e no computador na linguagem de programação. Essa fase requer muita prática.

Baseado na aplicação da metodologia, na sua primeira fase - Resolução de problemas, jogos de lógica fazem parte do ambiente. O HALYEN disponibiliza, até o momento, quatro jogos: Jogo da Sobrevivência, Jogo dos Maridos, A Grande Fuga e Jogo dos Vasos. Os jogos, desenvolvidos pela equipe em [41], foram adaptados neste projeto para coletar os dados necessários ao ambiente. A seguir, apresenta-se a descrição de um dos jogos.

\section{Jogo da Sobrevivência}

Descrição: existem cinco personagens presos em uma ilha. O aluno tem que transportá-los para a costa antes 
que o nível da água suba. O tempo para a tarefa é de trinta minutos. Na Figura 3 é apresentada a tela inicial do jogo.

Regras: para o transporte dos personagens, é utilizada uma jangada, que suporta apenas duas pessoas por vez. O tempo de travessia depende do peso dos personagens que estão na jangada.

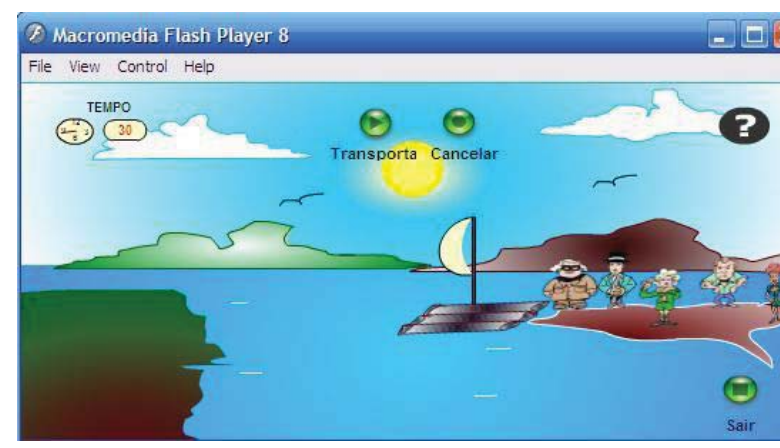

Figura 3: Tela inicial do Jogo da Sobrevivencia

Objetivo do jogo para o ensino de programação: desenvolver o raciocínio lógico dos alunos. Ajuda na formalização em forma algorítmica, pois a solução é intuitivamente exposta passo a passo.

Os jogos foram adaptados para registrar ações do jogador. Assim, o ambiente recolhe dados que representam a interação dos alunos e o acontecimento dos evento. Para cada jogo são armazenados as partidas, os movimentos do aluno no jogo baseado nos eventos estabelecidos, número de vezes que pede ajuda, etc. Foram definidos também eventos globais para os jogos tais como ganhar o jogo, perder o jogo, tempo real que o aluno demorou jogando uma partida, número de vitórias e derrotas. Para esse jogo foram recolhidos os seguintes dados:

- Tipo do movimento (Transportar ou Cancelar)

- Número de movimentos Transportar.

- Número de movimentos Cancelar.

- Número de vezes que pede ajuda.

- Tempo do jogo.

- Vitória no Jogo.

- Derrota no Jogo.

- Número de Vitórias.

- Número de Derrotas.

\section{Eventos e inferência das emoções}

Os eventos que podem vir a manifestar alguma das quatro emoções foram definidos. A Tabela 2 mostra os eventos identificados nos jogos. Cada emoção é disparada por um grupo de eventos. No entanto, a intensidade varia, sendo que um mesmo evento pode manifestar emoções diferentes, em intensidades diferentes.

O evento "o aluno perde o jogo" é analisado em dois momentos: o aluno não conseguiu concluir o jogo ou o jogo não teve uma solução correta. No caso do evento "Quantidade de partidas..." é calculado o número de tentativas até conseguir uma solução correta. O evento do aluno pedir ou aceitar ajuda sugerida pelo sistema é tratado de maneira diferenciada se o aluno é Orientado à aprendizagem ou a desempenho.

Alegria-tristeza, satisfação-frustração são emoções inferidas a partir da relação do aluno com a atividade. A abordagem de inferência das emoções é baseada nas propostas descritas em [40] e [42]. A inferência das emoções no ambiente é feita através de regras estruturadas do tipo IF-THEN e incluem vários parâmetros: a ocorrência de um evento, a desejabilidade de ter acontecido o evento, a manifestação da emoção e sua intensidade.

A manifestação de emoções é mensurada a partir da sua intensidade. Diante da ocorrência de um determinado evento, é analisada a desejabilidade do aluno em relação a esse evento e, dependendo do seu perfil e do estado emocional do aluno no momento de ocorrência do evento, será gerado o cálculo da intensidade de manifestação de uma emoção. No entanto, a emoção só se manifestará de fato caso esse valor seja igual ou ultrapasse o limiar mínimo de manifestação de emoção. A emoção que se manifestará é a que possuir maior valor de intensidade. Esta abordagem foi adaptada da proposta de [42].

Os jogos incorporam o cálculo do limiar mínimo de cada emoção. Para isto, na fase de coleta de dados (de treinamento) foram inseridas telas de interação, que coletam a emoção sentida no momento da ocorrência de um evento identificado e o nível de intensidade da mesma. Cinco níveis de intensidade para um evento foram estabelecidos para diferenciar a emoção sentida pelo aluno em um dado momento de tempo. Cada um desses níveis está associado a um valor na escala de 1 a 5 , sendo 1 o valor de menor intensidade. Dessa forma, os resultados irão corresponder à realidade do aluno no exato momento em que um evento aconteceu.

As emoções do aluno podem ser determinadas pelo cumprimento dos eventos a partir da sua orientação. $\mathrm{O}$ cumprimento correto de uma atividade, a resposta incorreta a um exercício, ou uma solicitação de ajuda são eventos, que relacionados à orientação do aluno, podem influenciar no processo de inferência de emoções. A orientação pode ajudar a mensurar a desejabilidade do evento: o quanto um aluno está afetado (positiva ou negativamente) com a ocorrência deste evento. Foram definidas algumas hipóteses para o modelo afetivo do aluno, que foram testadas com os alunos.

Essas hipóteses tentam generalizar a emoção do aluno e seu limiar de intensidade, a partir da ocorrência de determinados eventos e do perfil do aluno. Nesse sentido, as regras iniciais aplicadas no O HALYEN são mostradas 
a seguir com sete (7) casos específicos (IF-THEN).

\begin{tabular}{|c|c|}
\hline Emoção & Evento \\
\hline Alegria & $\begin{array}{l}\text { Ganhar o jogo } \\
\text { - O aluno conseguiu resolver o jogo corretamente }\end{array}$ \\
\hline Tristeza & $\begin{array}{l}\text { Perder o jogo } \\
\text { - O aluno não conseguiu concluir o jogo } \\
\text { - O jogo não teve uma solução correta }\end{array}$ \\
\hline Satisfação & $\begin{array}{l}\text { Ganhar o jogo } \\
\text { - O aluno conseguiu resolver o jogo corretamente } \\
\text { Tempo de realização menor que a média } \\
\text { Quantidade de partidas menor que a média } \\
\text { Quantidade de movimentos cancelados menor que a média (em uma partida) } \\
\text { Quantidade de passos igual ao número mínimo (solução ótima) }\end{array}$ \\
\hline Frustração & $\begin{array}{l}\text { Perder o jogo } \\
\text { - O aluno não conseguiu concluir o jogo } \\
\text { - O jogo não teve uma solução correta } \\
\text { Tempo de realização maior que a média } \\
\text { Quantidade de partidas maior que a média } \\
\text { Quantidade de movimentos cancelados maior que a média (em uma partida) } \\
\text { Quantidade de passos maior que o número mínimo } \\
\text { O aluno pediu ou aceitou ajuda (aluno voltado a desempenho) } \\
\text { - O aluno executa o assistente (de perguntas) }\end{array}$ \\
\hline
\end{tabular}

Tabela 2: Emoções e eventos associados

\section{Caso 1}

IF Evento: perdeu o jogo ^ aluno OD $\wedge$ desejabilidade = não THEN emoção: frustração ^ limiar de intensidade: 4

Estratégia proposta: mensagem de encorajamento.

\section{Caso 2}

IF Evento: perdeu o jogo pela primeira vez $\wedge$ aluno $O A \wedge$ desejabilidade = não THEN emoção: frustração $\wedge$ limiar de intensidade: 2

Estratégia proposta: mensagem de encorajamento e oferecer ajuda.

Caso 3

IF Evento: perdeu o jogo pela primeira vez aluno $\mathrm{OD} \wedge$ desejabilidade = não THEN emoção: frustração ^ limiar de intensidade: 4

Estratégia proposta: mensagem de encorajamento e oferecer ajuda.

\section{Caso 4}

IF Evento: chamou o assistente a dúvidas ^ aluno OD $\wedge$ desejabilidade = não THEN emoção: frustração ^limiar de intensidade: 2

Estratégia proposta: não fazer nada.

Caso 5

IF Evento: negou ajuda pela primeira vez ^ aluno $\mathrm{OD} \wedge$ desejabilidade = não THEN emoção: frustração ^ limiar de intensidade: 1

Estratégia proposta: mensagem "tem certeza?" + máximo de mais três tentativas sem interrupÇão.

Caso 6

IF Evento: pediu ajuda pela segunda vez ^ aluno

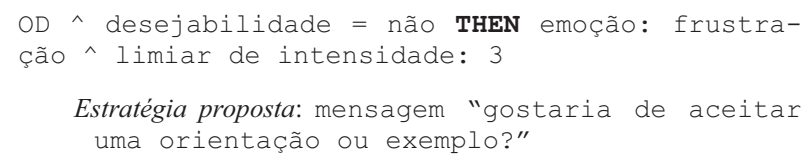

IF Evento: ganhou 0 jogo $\wedge$ desejabilidade $=$ sim THEN emoção: alegria ^ limiar de intensidade: 2, emoção: satisfação ^ limiar de intensidade: 5

Estratégia proposta: mensagem de congratulações

No Caso 7, acima, a emoção que se manifestará é a satisfação pois a intensidade tem maior valor. A maioria dos eventos definidos são eventos associados a emoções negativas, pois eventos associados a emoções positivas pressupõem um aumento da motivação ou incentivam o aluno para próximas atividades. O nosso foco principal é coletar dados sobre emoções que possam interferir no desenvolvimento do aprendizado.

\section{Resultados preliminares}

Os resultados deste trabalho são de dois tipos: o sistema O HALYEN e a utilização do sistema em sala de aula.

O desenvolvimento do sistema baseou-se no paradigma de Orientação a Objetos, é um sistema Web que usa a linguagem de programação Java e a tecnologia JSP (Java Server Pages). Os jogos de lógica fazem parte do sistema para sustentar o primeiro passo da metodologia: resolução de problemas. Os jogos foram adaptados dos originais, fornecidos pelos autores [41], e desenvolvidos em Flash MX. 
O sistema é um ambiente que implementa um STI utilizando agentes inteligentes. O sistema faz o cálculo do perfil do aluno. Possui o assistente a dúvidas e está preparado para inserir outros assistentes.

Para o trabalho com os agentes inteligentes, utilizouse o framework JADE [43]. Para visualização da comunicação entre os agentes e o funcionamento do ambiente, apresenta-se um cenário onde um aluno entra no ambiente e joga uma partida. Na Figura 4, as trocas de mensagens da linha 1 à 4 são referentes ao $\operatorname{login}$ do aluno. A ferramenta SnifferAgent do JADE mostra a troca de mensagens graficamente em notação similar a diagramas de sequências UML entre os agentes.

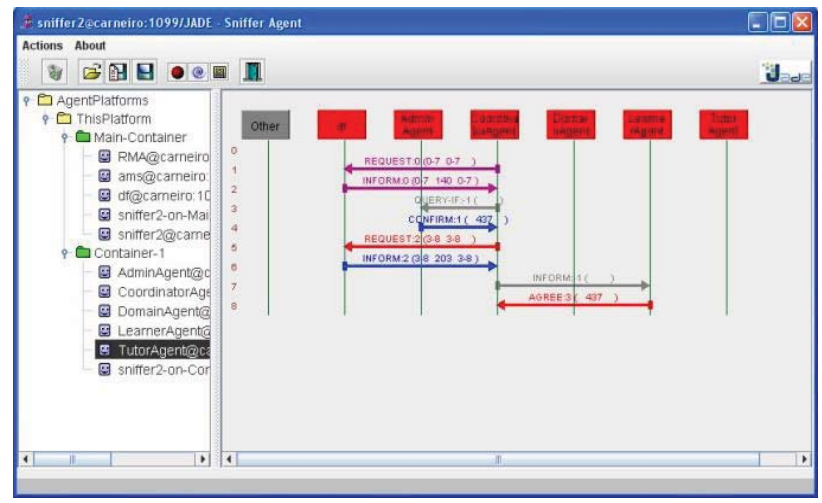

Figura 4: Fluxo de mensagens entre os agentes no cenário: login e joga

Detalhes da comunicação entre os agentes e o funcionamento do ambiente podem ser analisados no trabalho descrito em [29]. Enquanto o aluno está jogando, seus movimentos vão sendo gravados. Quando a partida é concluída, esse histórico é enviado para o Agente Coordenador, que por sua vez encaminha para o Agente Aprendiz. Este último armazena os dados referente à partida do aluno.

Quanto à utilização do ambiente em sala de aula, a fase de treinamento de O HALYEN foi executada com sucesso. Esta etapa teve como principal objetivo recolher, através de experimento real, os dados de alunos que estão em estágio inicial de aprendizado de uma linguagem de programação. Foram realizadas as seguintes tarefas:

- Preenchimento do questionário MLSQ (Figura 5);

- recolhimento de dados referentes aos jogos e as telas de interação;

- análise das emoções dos alunos;

- avaliação das estratégias pedagógicas por parte dos alunos;

- o ajuste do limiar mínimo das quatro emoções.

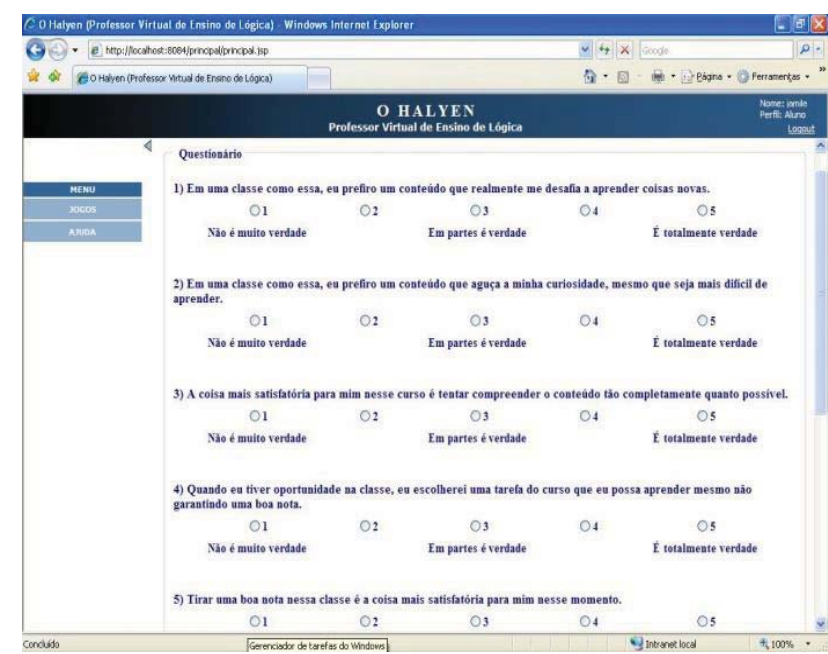

Figura 5: Tela para um preenchimento do questionário no primeiro acesso a O HALYEN

O ambiente foi disponibilizado para duas turmas de alunos de graduação do curso de Bacharelado em Ciência da Computação da UENF, durante as disciplinas de Programação I e Estrutura de Dados I, pertencentes ao primeiro e terceiro semestre do curso, respectivamente. Trinta e três (33) alunos participaram da oficina, sete mulheres e vinte e seis homens. A oficina foi proposta para os alunos na forma de atividade não avaliativa (não valia nota para a disciplina) e voluntária. Mesmo assim, todos os alunos das duas turmas aceitaram executá-la. Inicialmente, houve um breve treinamento sobre o funcionamento do ambiente e os alunos rapidamente aprenderam a manuseá-lo.

A Tabela 3 mostra a classificação dos alunos entre os dois perfis iniciais, calculados a partir do formulário MSLQ: $57,14 \%$ tem orientação extrínseca ou é Orientado ao desempenho e $37,14 \%$ tem orientação intrínseca ou é aluno Orientado à aprendizagem.

\begin{tabular}{|l|c|}
\hline \multicolumn{1}{|c|}{ PERFIL INICIAL } & \\
\hline Orientado à aprendizagem & 20 \\
\hline Orientado ao desempenho & 13 \\
\hline \multicolumn{2}{|c|}{ TOTAL DE ALUNOS }
\end{tabular}

Tabela 3. Distribuição dos alunos pelos perfis

O experimento consistiu em apresentar para os estudantes os quatro jogos de lógica com diferentes níveis de complexidade no contexto do ambiente virtual. Durante a atividade dos jogos, os estudantes descreveram seu estado afetivo antes de receber as ações pedagógicas e logo depois do evento acontecer. Ao acontecer o evento, foi coletada a desejabilidade do evento, as emoções e sua intensidade.

A lista de eventos que ativaram a coleta de dados so- 
bre as emoções é mostrada na Tabela 4. Os eventos correspondem às hipóteses expostas na seção anterior. Assim, se o aluno precisou solicitar ajuda para resolver o jogo ou precisou de mais de uma partida para solucionar o jogo corretamente, a interface (de interação) foi ativada para coleta da intensidade da emoção.

\section{Lista de eventos quanto à emoção}

(Valência)

\begin{tabular}{|l|l|}
\hline Perder o jogo & Negativo \\
Ganhar o jogo & Positivo \\
\hline $\begin{array}{l}\text { Não conseguir completar a partida } \\
\text { Precisar solicitar ajuda durante o } \\
\text { jogo }\end{array}$ & Negativo \\
\hline $\begin{array}{l}\text { Precisar solicitar ajuda pela se- } \\
\text { gunda vez durante o jogo }\end{array}$ & Negativo \\
$\begin{array}{l}\text { Aceitar ajuda durante o jogo } \\
\text { Precisar de mais de uma partida } \\
\text { para resolver o jogo }\end{array}$ & Negativo \\
\hline
\end{tabular}

Tabela 4. Eventos associados ao estado emocional do aluno

A primeira análise feita sobre os resultados obtidos foi fazer uma varredura para detectar inconsistências nas respostas dos alunos. O resultado da varredura foi bem sucedido, sendo que não houve nenhum aluno com valores contraditórios.

$\mathrm{Na}$ Tabela 5, são mostrados os resultados da coleta de dados. Para cada evento, foram consideradas as duas emoções que mais se manifestaram, na escala de intensidade de 1 a 5, por classe de aluno (orientado ao desempenho ou à aprendizagem). Os resultados em negrito indicam a intensidade onde se encontra a maioria dos alunos de cada classe para o par evento/emoção. Logo, observase que para o evento "não conseguir completar uma partida do jogo", 60\% dos alunos orientados à aprendizagem não ficaram tristes o que quer dizer que esta emoção se manifestou com intensidade 1 ou pode-se interpretar que não houve manifestação de tristeza.

Observando o evento "ganhar o jogo", tem-se que a maioria dos alunos das duas classes, que conseguiram completar a atividade, manifestaram as emoções satisfação e alegria com intensidade máxima. A emoção de satisfação foi mais significativa.

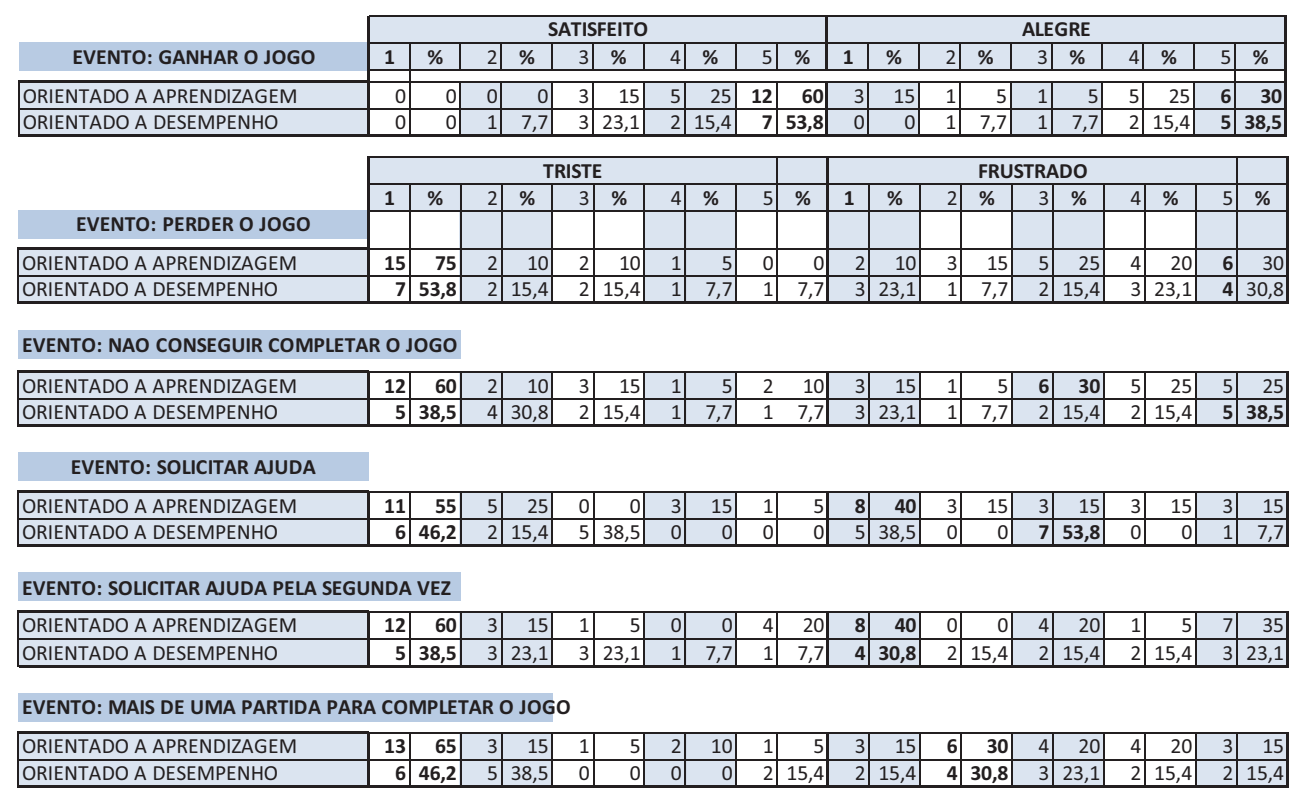

Tabela 5. Resultados sumarizados da coleta de dados

Adicionalmente, observa-se que todo aluno ao ganhar o jogo manifestou satisfação de nível de intensidade maior ou igual a três (3). A média ponderada das duas emoções na ocorrência desse evento foi maior que três, sendo que na média a satisfação predominou (maior que 4). Este resultado é compatível com o modelo apresenta- do em [40].

Observa-se que, de todos os eventos que podem acarretar emoções negativas em relação à atividade, e que requerem estratégias pedagógicas de incentivo e motivação, o evento "aceitar ajuda durante o jogo" não foi inclu- 
ído nas Tabelas 5 e 6. Isto, porque as emoções tristeza e frustração se manifestaram minimamente com intensidade 1 para todos os alunos de ambas as classes.

No restante dos eventos, a tristeza se manifestou com intensidade 1, passando assim a ser mais valiosa a frustração para tomar ação pedagógica. Sendo que nos eventos de perder o jogo ou não conseguir completá-lo ela se manifestou mais forte para a maioria com intensidade 5 . Destaca-se que para os alunos orientados ao desempenho, o fato de não conseguir completar o jogo foi mais importante que para os alunos orientados à aprendizagem. Porém, observaram-se três alunos OD que ficaram pouco frustrados e supõe-se a possibilidade desses alunos serem orientados a conseguir a nota mínima para passar, o que não é raro de acontecer com alguns alunos por turma.

Para os eventos de solicitação de ajuda, a maioria dos alunos OA manifestaram tristeza com baixa intensidade (menor ou igual a 2) e com frustração em todos os níveis predominando a intensidade mínima. Os alunos OD se declararam frustrados e tristes com intensidade menor e igual a 3 na ocorrência do evento. $O$ fato de solicitar ajuda pela segunda vez aumentou a intensidade da frustração para o aluno orientado à desempenho. Este resultado, também, é compatível com o modelo apresentado em [40].

Precisar de mais de uma partida para vencer o jogo não foi relevante em termos de tristeza para a maioria dos alunos (vinte e sete alunos com intensidade menor ou igual a 2). 69,3\% dos alunos OD ficaram frustrados com intensidade menor ou igual a três. Interpreta-se que o fato de vencer em mais de uma partida não afeta afetivamente de maneira relevante. Já os alunos OA foram mais versáteis na intensidade da frustração.

$\mathrm{Na}$ análise final dos dados utilizou-se da estatística descritiva com a média ponderada, baseada na fórmula

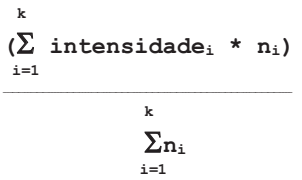

sendo $\mathbf{k}$ o número de níveis de intensidade definidos, isto é igual a cinco (5), intensidade é o nível de intensidade da emoção e $\mathbf{n}_{i}$ o número de alunos que manifestou esse nível de emoção.

Também, foi realizada uma análise da dispersão da intensidade por meio do cálculo da entropia da informação. A entropia da informação caracteriza a (im)pureza de uma coleção de amostras e é definida pela fórmula

$$
e\left(d_{i}\right)=-\alpha\left(\sum_{j=1}^{k}-p_{i}^{j} \ln \left(p_{i}^{j}\right)\right)
$$

onde $\mathrm{p}_{i}^{j}=\mathrm{n}_{\mathrm{i}}^{j} \mathrm{t}_{\mathrm{i}}$. O termo $\mathrm{n}_{\mathrm{i}}^{j}$ é o número de alunos que manifestou o nível de emoção na intensidade $j$ para o evento $i$ e $t_{i}$ é o tamanho da amostra (total de alunos distribuídos pelos diferentes níveis de intensidade para o evento i). O termo $\alpha=1 / \ln (\mathrm{k}), \quad \alpha>0$ é um fator de normalização para $\mathbf{k}$ níveis de intensidade. A entropia da informação resulta em valores entre 0 e 1 . Quanto mais próximo de zero mais pura a amostra é, quanto mais próximo de 1 mais impureza tem.

A Tabela 6 mostra a média ponderada e a entropia calculadas a partir dos valores da Tabela 5. Como se observa na Tabela 6, em geral, a intensidade das emoções variou razoavelmente para os eventos, o que significa que o conjunto amostral se mostrou com alto nível de impureza. Porém, os valores de entropia da tristeza para perder o jogo (para alunos OA) e a frustração por pedir ajuda (para alunos $\mathrm{OD})$ tiveram as entropias mais baixas $(0.51 \mathrm{e}$ 0.56 , respectivamente), o que significa que o número de alunos ficou concentrado em poucos níveis de intensidade (1 para primeiro caso, e 1 e 3 para o segundo). Esses resultados são compatíveis com o trabalho descrito em [40].

A proposta a partir desta análise é definir o limiar de intensidade de emoções para os eventos considerados. O limiar é calculado como a parte inteira da média ponderada. Logo, para um aluno orientado à aprendizagem o limiar de intensidade para a inferência da emoção frustração diante o evento "perder o jogo" é igual a 3, desde que essa seja a emoção que mais se manifeste (de maior intensidade para o evento). Para poder obter os limiares das emoções do modelo afetivo do aluno, os estudantes responderam as telas de interação quando determinados eventos aconteceram.

Por último, todos os alunos conseguiram resolver os jogos durante a oficina. O número médio de partidas por aluno para resolver todos os jogos foi de 4 partidas e nenhum aluno conseguiu solucionar corretamente o jogo na primeira partida.

\subsection{Avaliação das estratégias pedagógicas}

Após a oficina, foi solicitado para todos os alunos avaliarem as estratégias pedagógicas. Os alunos tiveram que responder se as estratégias pedagógicas foram adequadas e se ajudaram no processo de aprendizagem.

As ações pedagógicas foram focadas para as emoções de tristeza e frustração. A Tabela 7 mostra as estratégias pedagógicas definidas para o ambiente. 


\begin{tabular}{|c|c|c|c|c|}
\hline \multirow[b]{3}{*}{ EVENTO: GANHAR O JOGO } & \multicolumn{4}{|c|}{ EMOÇÕES } \\
\hline & \multicolumn{2}{|c|}{ SATISFEITO } & \multicolumn{2}{|c|}{ ALEGRE } \\
\hline & Média & Entropia & Média & Entropia \\
\hline ORIENTADO A APRENDIZAGEM & 4,45 & 0,58 & 3,50 & 0,80 \\
\hline \multirow[t]{3}{*}{ ORIENTADO A DESEMPENHO } & 4,15 & 0,72 & 3,85 & 0,65 \\
\hline & \multicolumn{4}{|c|}{ EMOÇÕES } \\
\hline & \multicolumn{2}{|c|}{ TRISTE } & \multicolumn{2}{|c|}{ FRUSTRADO } \\
\hline EVENTO: PERDER O JOGO & Média & Entropia & Média & Entropia \\
\hline ORIENTADO A APRENDIZAGEM & 1,45 & 0,51 & 3,45 & 0,96 \\
\hline ORIENTADO A DESEMPENHO & 2,00 & 0,81 & 3,31 & 0,95 \\
\hline
\end{tabular}

EVENTO: NAO CONSEGUIR COMPLETAR O JOGO

\begin{tabular}{|l|r|r|r|r|}
\hline ORIENTADO A APRENDIZAGEM & 1,95 & 0,75 & 3,40 & 0,92 \\
\hline ORIENTADO A DESEMPENHO & 2,15 & 0,88 & 3,38 & 0,92 \\
\hline
\end{tabular}

\section{EVENTO: SOLICITAR AJUDA}

\begin{tabular}{|l|r|r|r|r|}
\hline ORIENTADO A APRENDIZAGEM & 1,90 & 0,69 & 2,50 & 0,93 \\
\hline ORIENTADO A DESEMPENHO & 1,92 & 0,63 & 2,38 & $\mathbf{0 , 5 6}$ \\
\hline
\end{tabular}

\section{EVENTO: SOLICITAR AJUDA PELA SEGUNDA VEZ}

\begin{tabular}{|l|r|r|r|r|}
\hline ORIENTADO A APRENDIZAGEM & 2,05 & 0,66 & 2,95 & 0,75 \\
\hline ORIENTADO A DESEMPENHO & 2,23 & 0,89 & 2,85 & 0,61 \\
\hline
\end{tabular}

EVENTO: MAIS DE UMA PARTIDA PARA COMPLETAR O JOGO

ORIENTADO A APRENDIZAGEM ORIENTADO A DESEMPENHO

Tabela 6. Estatística descritiva e entropia

\section{Ações Pedagógicas}

A- Quando você perdeu o jogo, foi uma estratégia aceitável o sistema lhe enviar uma mensagem de encorajamento?

B- Quando você perdeu o jogo pela terceira vez, foi uma estratégia aceitável o sistema lhe enviar uma mensagem de encorajamento e lhe oferecer ajuda?

C- Ao você não aceitar ajuda na primeira vez oferecida, foi uma estratégia aceitável o sistema lhe enviar uma mensagem de "tem certeza?"?

D- Ao você não aceitar ajuda na primeira vez oferecida, foi uma estratégia aceitável o sistema cuidar de não insistir, dando, no máximo, três tentativas sem interrupção?

E- Ao você não aceitar ajuda na segunda vez oferecida, foi uma estratégia aceitável o sistema lhe enviar uma mensagem "gostaria de aceitar uma orientação ou exemplo?"?

Tabela 7. Estratégias pedagógicas

A Tabela 8 ilustra os resultados obtidos classificados para os alunos dos dois perfis. Com os resultados mostrados na Tabela 8 , pode-se concluir que as estratégias em geral foram bem aceitas pelos alunos. No caso da estratégia "Ao você não aceitar ajuda na primeira vez oferecida, teria sido uma estratégia aceitável o sistema lhe enviar uma mensagem de "tem certeza?"?" não se mostrou útil para o $76 \%$ dos alunos.

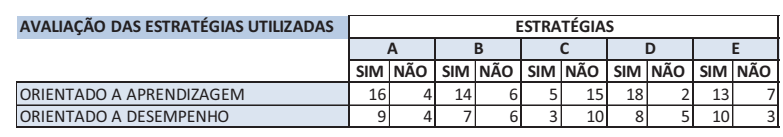

Tabela 8. Avaliação das estratégias por parte dos alunos

O gráfico da Figura 6 mostra, para cada estratégia, as respostas positivas (barra inferior) e as respostas negativas (barra superior). Para a maioria dos alunos, as estra- tégias A, B, D e E se mostraram úteis. No tocante aos alunos OA, observa-se a aprovação de $65 \%$ (Estratégia E) a 90\% (Estratégia D). Com respeito aos alunos OD, 54\% (Estratégia B) a 77\% (Estratégia E) concordaram com essas estratégias propostas.

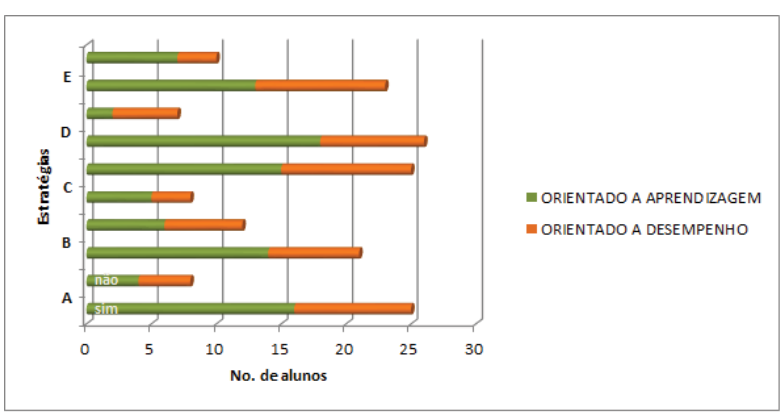

Figura 6: Gráfico da avaliação das estratégias propostas

\section{Considerações Finais}

A contribuição principal do artigo é a aplicação da metodologia de ensino auxiliada pelos recursos tecnológicos, que apoiam o desenvolvimento de habilidades dos alunos na aprendizagem de programação. Os recursos tecnológicos foram integrados em um sistema tutor inteligente. A criação do sistema O HALYEN como um ambiente virtual em duas fases permitiu coletar dados sobre perfis dos alunos e emoções na primeira fase para uma posterior aplicação do conhecimento aprendido na segunda.

A nossa experiência até o momento tem sido muito animadora. As questões introduzidas neste artigo: $a$ inclusão de recursos tecnológicos no ambiente de aprendizagem é efetiva para auxiliar o trabalho em sala de aula? a inferência das emoções dos alunos utilizando recursos tecnológicos no ambiente de aprendizagem pode influenciar positivamente no aprendizado dos alunos? foram respondidas com os resultados apresentados, pois a avaliação por parte dos alunos das ações pedagógicas dado o acontecimento dos eventos foi positiva (as ações foram consideradas úteis). Porém, os resultados não são concluintes, já que o número de alunos que participou da oficina considera-se pequeno.

Nessa primeira oficina, observou-se que os alunos ficam motivados com a abordagem de uso dos jogos, e que os alunos tendem a numerar os passos das soluções e abreviar os nomes dos objetos dos jogos. Isto faz com que os jogos se mostrem muito úteis na formalização de algoritmos.

As hipóteses estabelecidas foram verificadas. De fato, as emoções esperadas para cada evento foram as emoções manifestadas pelos alunos para a maioria dos casos.

A integração da metodologia com recursos tecnológi- 
cos que apoiem o desenvolvimento de habilidades dos alunos na aprendizagem de programação parece muito boa e promissora. No entanto, a necessidade de formação de oficinas é de muita importância para continuar a validar o ambiente e a proposta, assim como para a acumulação de dados que permitam obter bons resultados na classificação dos perfis dos alunos.

Outras questões do MSLQ para ajudar a determinar o perfil do aluno serão inseridas no ambiente. Os assistentes inteligentes são uma contribuição importante para apoiar o desenvolvimento e a interatividade dos alunos dentro do ambiente. Está sendo desenvolvido um agente pedagógico animado para interagir com o aluno. A inferência das emoções será feita e validada a partir dos limiares mínimos definidos como parte dos resultados. A inclusão de outras emoções do modelo OCC está sendo planejada.

\section{Agradecimentos}

Esta pesquisa contou com o apoio financeiro da Fundação Carlos Chagas Filho de Amparo à Pesquisa do Estado do Rio de Janeiro (FAPERJ) e do CNPq.

\section{Referências}

[1] R. L. B. L. Campos. Metodologia ERM2C: Para melhoria do processo de ensino-aprendizagem de lógica de programação. In Anais do XVIII WEI - Workshop sobre Educação em Computação, 2010.

[2] F. Alexandrini, J.E. de Fáveri, Th.S. Araujo, C.F.D. Alexandrini. Estudo de Caso do Processo de Ensino-Aprendizagem de Introdução à Informática e Programação em IES Comunitária de Santa Catarina. In Anais do VII Simpósio de Excelência em Gestão e Tecnologia - SEGeT, 2010.

[3] M. C. Rodrigues Jr. Experiências Positivas para o Ensino de Algoritmos. In Anais do XII WEI Workshop de Educação em Computação, 2004.

[4] H. Hostins e A. Raabe. Auxiliando a aprendizagem de algoritmos com a ferramenta WEBPORTUGOL. In Anais do XV WEI - Workshop sobre Educação em Computação, 2007.

[5] M. P. Mota, L. W. K. Pereira, E. L. Favero. JAVATOOL: Uma ferramenta para ensino de programação. In Anais do XVI WEI - Workshop sobre Educação em Computação, 2008.

[6] J. C. R. Pereira Jr., C.E. Rapkiewicz, C. Delgado, J.A.M. Xexéo. Ensino de algoritmos e programação: uma experiência no nível médio. In
Anais do X WEI - Workshop de Educação em Informática, 2005.

[7] S. M. González e A. R. Tamariz. Uma experiência no ensino de programação para cursos de Engenharia. In Anais do XXVIII Encontro Nacional de Engenharia de Produção. RJ, 2008.

[8] C. Delgado, J.A.M. Xexéo, I. F. de SOUZA, M. Campos, C.E. Rapkiewicz. Uma abordagem pedagógica para iniciação ao estudo de algoritmos. In Anais do IX WEI - Workshop de Educação em Informática, 2004.

[9] J. Moreno. Uso de juegos digitales educativos como herramienta de soporte para el aprendizaje de algoritmos. Revista Novas Tecnologías na Educação (RENOTE). CINTED-UFRGS. Vol. 8 , No. 3. 2010.

[10] B. Bodenheimer, B. Williams, M. R. Kramer, K. Viswanath, R. Balachandran, K. Belynne, G. Biswas. Construction and Evaluation of Animated Teachable Agents. Educational Technology \& Society. 12(3): 191- 205, 2009.

[11] Y. H. Pérez, L. E. S. Succar, G. A. Figueroa. Desarrollo e Integración de un Modelo de Comportamiento Afectivo a un Sistema Tutor Inteligente. Revista Iberoamericana de Tecnologías del Aprendizaje (IEEE-RITA). 2(4): 161-169, 2009

[12] E. C. M. Tassoni, S. A. S. Leite. A relação afeto, cognição e práticas pedagógicas. In Anais da $33^{a}$ Reunião Anual da ANPEd. Educação no Brasil: o balanço de uma década, 2010.

[13] L. M. L. Rodrigues, M. Carvalho. STI-I: Sistemas Tutoriais Inteligentes que integram Cognição, Emoção e Motivação. Revista Brasileira de Informática na Educação (RBIE). 13(1), 2005.

[14] R. M. Vicari, L. M. Giraffa. Fundamentos dos Sistemas Tutores Inteligentes. Sociedades artificiais: a nova fronteira da inteligência nas máquinas. Porto Alegre: Bookman. Pag. 155-208, Vol. 1, 2003.

[15] M. Arevalillo-Herráez, D. Arnau, L. M. Giménez, Domain-specific knowledge representation and inference engine for an intelligent tutoring system, Knowledge-Based Systems, Vol. 49, Pag. 97-105, September 2013.

[16] Sheng-Jen Hsieh, Yuan-Teng Cheng. Algorithm and intelligent tutoring system design for programmable controller programming. The International Journal of Advanced Manufacturing 
Technology. 71(5), -8, Pag. 1099-1115, 2014

[17] L. Zhang, K. VanLehn, S. Girard, W. Burleson, M.E. Chavez-Echeagaray, J. González-Sánchez, Y. Hidalgo-Pontet. Evaluation of a meta-tutor for constructing models of dynamic systems, Computers \& Education, Vol. 75 Pag. 196-217, June 2014.

[18] K. Chrysafiadi, M. Virvou. Evaluating the integration of fuzzy logic into the student model of a web-based learning environment. Expert Systems with Applications, 39(18) , Pag. 13127-13134, 15 December 2012.

[19] Z. Jeremic; J. Jovanovic; D. Gasevic. Evaluating an Intelligent Tutoring System for Design Patterns: The DEPTHS Experience. Educational Technology \& Society, 12(2), Pag.111-130, 2009.

[20] R.S.J.d. Baker, S.K. D’Mello, M.M.T. Rodrigo, A.C. Graesser. Better to be frustrated than bored: The incidence, persistence and impact of learners'cognitive- affective states during interactions with three different computer-based learning environments. Int. J. Human-Computer Studies. Elsevier. 68(1): 223-241. 2010.

[21] T. Castro, H. Fuks, M. A. F. Spósito, A. de Castro Jr. Análise de um Estudo de Caso para Aprendizagem de Programação em Grupo. Revista Iberoamericana de Tecnologías del Aprendizaje (IEEE-RITA). 4(2):155-160. 2009.

[22] L. F. Dias Pereira, F. R. Lapolli, F.F. Sampaio, Cl.L.R. Motta, C.E. Tolla de Oliveira. Ateliê de Objetos de Aprendizagem : Uma Abordagem para o Ensino de Computação em Cursos Técnicos. Revista Brasileira de Informática na Educação (RBIE). 18(3). 2010.

[23] S. W. Martins, A.J. Mendes, A.D. Figueiredo. Comunidades de Investigação em Programação: Uma Estratégia de Apoio ao Aprendizado Inicial de Programação. Revista Iberoamericana de Tecnologías del Aprendizaje (IEEE-RITA). 5(1): 39-46. 2010.

[24] L. Giraffa, S.S. Marczak, G. Almeida. O ensino de algoritmos e programação mediado por um ambiente Web. In Anais do XXIII Congresso da Sociedade Brasileira de Computação (SBC). UNICAMP, Campinas, Brasil. 2003

[25] E. R. Sykes e F. Franek. An intelligent tutoring system prototype for learning to program Java. In Proceedings of $3^{\text {rd }}$ IEEE International Conference on Advanced Learning Technologies.
Atenas, Grécia, 2003.

[26] P. Brusilovsky, E. Schwarz, G. Weber. ELMART: An Intelligent Tutoring System on World Wide Web. In Intelligent Tutoring Systems (Lecture Notes in Computer Science, Vol. 1086). Berlin: Springer Verlag. Pag. 261-269. 1996.

[27] A. R. Tamariz, S. M. González, C.B. Pessanha, E.C. Braga. Emoções como parte de um ambiente virtual de aprendizagem. In Proceedings of the Ibero Americana Conference WWW/Internet (IADIS), Vila Real, Portugal. 2007.

[28] A. R. Tamariz, E. H. de Almeida Gomes, S. M. González. Proposta de um modelo cognitivo para o ambiente virtual de aprendizagem: $\mathrm{O}$ HALYEN. In Proceedings of the Ibero Americana Conference WWW/Internet (IADIS), Alcalá de Henares - Madrid, Espanha. 2009.

[29] S. M. González, A. R. Tamariz, E. C. Carneiro, J.S. de Almeida. Agentes Inteligentes no ambiente virtual de ensino de lógica HALYEN. In Proceedings of the Ibero Americana Conference WWW/Internet (IADIS), Vila Real, Portugal. 2007.

[30] Yacef, K. (2002). Intelligent teaching assistant systems. Proceedings of the IEEE International Conference on Computers in Education, volume 1, pages 136-140, New Zeland.

[31] E. Moura, S.M. González, A.R. Tamariz. Uma ferramenta de mineração de textos para assistir as dúvidas dos alunos em ambientes virtuais de aprendizagem. Proceedings of the Ibero Americana Conference WWW/Internet (IADIS), Vila Real, Portugal. 2007.

[32] P. A. Jacques, R. M. Vicari. PAT: um agente pedagógico animado para interagir afetivamente com o aluno. CINTED: Novas Tecnologias na Educação, UFRGS, Maio, 2005.

[33] A. Orthony, G. L. Clore, A. Collins. The Cognitive Structure of Emotions. Cambridge University Press, Cambridge, UK. 1988.

[34] A. de Vicente, H. Pain. A computational model of affective educational dialogues. Papers from the 2000 Fall Symposium: Building Dialog Systems for Tutorial Applications. North Falmouth, Massachusetts, November 3-5, 2000. Technical Report FS-00-01, AAAI Press, Menlo Park, CA, USA. pp 113-121, 2000.

[35] M. T. Longhi, M. Bercht, P. A. Behar. O desafio de reconhecer a dimensão afetiva em ambiente Virtual de aprendizagem. In Anais do XIX Sim- 
pósio Brasileiro de Informática na Educação (SBIE). 2008.

[36] M. C. Rinaudo, A. Chiecher, D. S. Donolo. Motivación y uso de estrategias en estudiantes universitarios. Su evaluación a partir del Motivated Strategies Learning Questionnaire. Revista Anales de Psicología. 19(1):107-119. 2003.

[37] M. C. Rinaudo, M. L. de la Barrera, D. S. Donolo. Motivación para el aprendizaje en alumnos universitarios. Revista Electrónica de Motivación y Emoción (REME). 9(22): 107-119. 2006.

[38] P. R. Pintrich, D. A. F. Smith, T. Garcia, W. J. McKeachie. A Manual for the Use of the Motivated Strategies for Learning Questionnaire. University of Michigan, TR 91-B-004, 1991.

[39] R. T. Taylor. Review of the Motivated Strategies for Learning Questionnaire (MSLQ) Using Reliability Generalization Techniques to Assess Scale Reliability, PhD Thesis, Auburn University, 2012 .

[40] P. A. Jaques, R. M. Vicari. A BDI approach to infer student's emotions in an intelligent learning environment, Computers \& Education, 49(2): 360-384. September 2007.

[41] N. Santana dos Santos, C.E. Rapkiewicz. Jogos Educacionais - ferramentas para o ensino de programação. Anais do I WEI TCHË- Workshop sobre Educação em Informática. 2007.

[42] D. F. Adamatti, A. L. C. Bazzan. AFRODITE: A Framework for Simulation of Agents with Emotions. Agent Based Simulation, Montpelier, Franca. 2003.

[43] V. Gyurjyan, D. Abbott, G. Heyes, E. Jastrzembski, C. Timmer, E. Wolin. FIPA agent based network distributed control system. In Proceedings of Computing in High Energy and Nuclear Physics. 2003. 